\title{
In die digitale Welt
}

\section{Liebe Leserinnen und Leser,}

Controller können sich nicht über zu wenig Arbeit beklagen. Ständig werden neue Themen an sie herangetragen, von Veränderungen in den IFRS über neue Inhalte wie Nachhaltigkeit bis hin zu neuen Rollen, wie etwa dem Data Scientist. Da ist es nur zu verständlich, bewährten Instrumenten zu vertrauen und nicht auch an dieser Front neue Baustellen zu eröffnen. Eines der wichtigsten „Arbeitspferde“ der Controller ist die Kostenrechnung. Als „Spinne im Netz“ unterstützt sie die Controller bei diversen Aufgaben des Tagesgeschäfts, von der operativen Planung bis zur monatlichen Berichterstattung, von der Ermittlung von Kundenergebnissen bis zur Bestimmung zulässiger steuerlicher Verrechnungspreise. Grund, an ihr zu zweifeln, besteht auch deshalb nicht, weil von Manager-Seite kein Anstoß kommt, sei es, weil die Manager zufrieden sind, sei es, dass sie die Qualität der Kostenrechnung nicht wirklich beurteilen können.

Bei genauem Hinsehen ist die Qualität der Kostenrechnung schleichend schlechter geworden. Dies sieht man beispielsweise an den eklatanten Fehlern in der Variantenkalkulation in vielen Unternehmen oder an der mangelnden Qualität von Durchschnittswerten, die die Kostenrechnung vielfältig durchziehen: Die Komplexität der Abläufe nimmt zu, ebenso wie deren Dynamik. Beides erhöht die Varianz. Die Kostenrechnung kennt aber nur Erwartungswerte. Diese passen immer schlechter auf den Einzelfall. Diese Entwicklung ist den meisten Controllern verborgen geblieben, auch deshalb, weil die Kostenrechnung - anders als die Finanzbuchhaltung keiner laufenden Qualitätskontrolle unterworfen ist.

Wenn heute in vielen Unternehmen wieder über Kostenrechnung nachgedacht wird, liegt dies nicht an einer Unzufriedenheit mit der Qualität des Instruments, sondern daran, dass die Systembasis, auf der sie läuft, vor einer grundlegenden Veränderung steht. Der anstehende Release-Wechsel von SAP R/3 zu S/4HANA macht ein Überdenken unausweichlich. Dieser Anstoß kann segensreich sein oder aber auch gefährlich. Segensreich dann, wenn die schöne neue Welt von gelösten sachlichen und zeitlichen Konsistenzproblemen, hoher Flexibilität und Realtime-Zugang zu den gesamten erfassten Daten dazu ge-

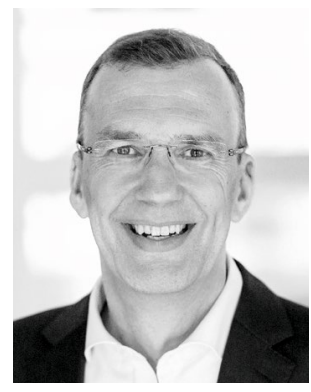

Utz Schäffer

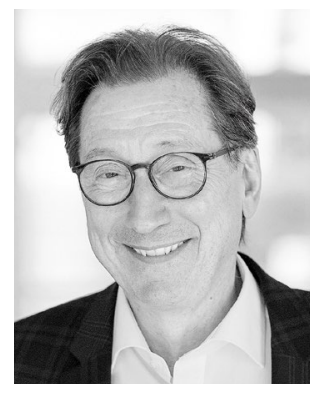

Jürgen Weber nutzt wird, die Qualität der Ausgangsdaten deutlich zu verbessern und auch die ganze Kostenrechnung auf den Prüfstand zu stellen. Gefährlich dann, wenn das Ziel nur darin besteht, das alte Konzept möglichst aufwandsarm und geräuschlos auf die neue Systemebene zu übertragen. Der Reflex für Letzteres ist häufig zu beobachten. Man scheut den ganz erheblichen Aufwand dafür, „reinen Tisch“ zu machen, Aufwand nicht nur für Controller, sondern auch für Manager, ohne die das nicht geht. Nur wer grundsätzlich über die Kostenrechnung nachdenkt, wird ihre Chancen in einem neuen, digitalen Kontext erkennen. Viele bisherige Begrenzungen fallen weg, jeder Einzelfall kann berücksichtigt werden, ohne ihn zu einem Durchschnitt zu verschmieren. Die oft geforderte Verbindung von finanziellen und nichtfinanziellen Steuerungsgrößen wird auf eine hochwertige Datenbasis gestellt. Auswertungen können zu jedem gewünschten Zeitpunkt durchgeführt werden - und die Liste ließe sich noch fast beliebig verlängern. Unser Plädoyer lautet also: Gehen Sie den Weg in die neue Kostenrechnungswelt, aber gehen Sie ihn erst, wenn Sie eine klare inhaltliche Vorstellung davon haben, wo Sie ankommen wollen!

Viel Spaß bei der Lektüre wünschen Ihnen
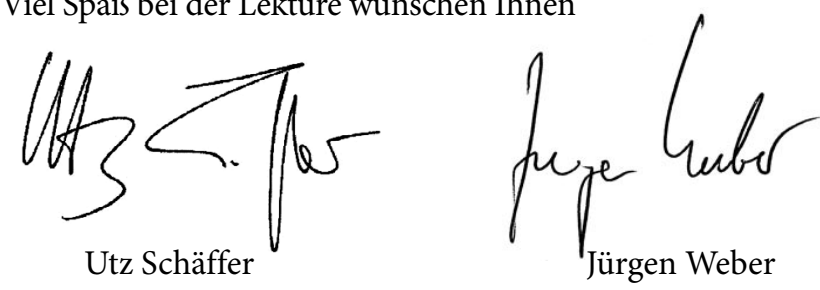